\title{
Phytotherapie
}

\section{Cholesterinsenker Königsartischocke}

- Immer mehr Menschen vertrauen auf die Heilkraft von Pflanzenarzneimitteln. Bei der ärztlichen Verordnung auf dem grünen Rezept und in der Selbstmedikation spielen sie deshalb eine große Rolle.,Moderne Phytopharmaka überzeugen nicht nur durch ihre Wirksamkeit, sondern auch durch ihre gute Verträglichkeit", so Prof. Dr. rer. nat. Alexander Schenk, Münster.

„Das deutsche Arzneibuch stellt hohe Anforderungen an die Qualität von Pflanzen, die zum Herstellen von Medikamenten genutzt werden", betonte Schenk. „Hochwertige pflanzliche Fertigarzneimittel sind das Ergebnis langjähriger Forschungsarbeit." Als Beispiel nannte Schenk Spezialextrakt aus der Königsartischocke, einer speziell für medizinische Zwecke gezüchteten Hochleistungssorte. "Meist haben Völlegefühl, Blähungen und Magendrücken ihre Ursache in Problemen der Fettverdauung", erklärte Schenk. Nach der Einnahme von Hepar-SL ${ }^{\circledR}$ forte Kapseln mit Königsartischocken-Spezialextrakt kann

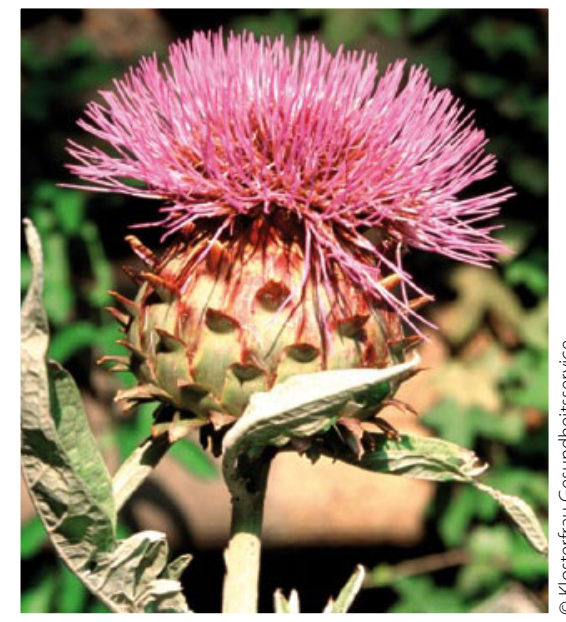

Königsartischocke (Cynara scolymus) hilft schnell bei dyspeptischen Beschwerden.

die Gallensaftproduktion bereits nach kurzer Zeit um mehr als das Doppelte gesteigert werden. Zudem entkrampfen die Wirkstoffe die Gallenwege, beugen Gallensteinen vor, regen die Peristaltik an und senken das LDL-Cholesterin um bis zu $20 \%$.
Die gelatine- und glutenfreien sowie ohne Konservierungsstoffe hergestellten Biocaps $^{\circledast}$ lösen sich im Magen sofort auf. Daher setzt ihre Wirkung meist schon nach wenigen Minuten ein.

In einer aktuellen Praxisstudie mit 118 Patienten mit dyspeptischen Beschwerden besserten täglich zwei Kapseln Spezialextrakt die Beschwerden schnell und deutlich. Am Ende der Studie, nach rund sechs Wochen, war die Intensität der funktionellen dyspeptischen Beschwerden erheblich gelindert. Vor allem Druck- und Völlegefühl sowie Blähungen und Oberbauchschmerzen besserten sich signifikant. Die klinische Wirkung wurde von $88 \%$ der Ärzte und $84 \%$ der Patienten mit "gut" bis "sehr gut" beurteilt, die Verträglichkeit von Ärzten und Patienten

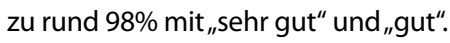

- Red.

Quelle: Fortbildungsveranstaltung „Homöopathie trifft Phytotherapie" der Niedersächsischen Akademie für Homöopathie und Naturheilverfahren, Celle, Juni 2011; Nach Informationen von Klosterfrau

\section{Strontiumranelat bei postmenopausaler Osteoporose \\ Biopsie-Studie belegt knochenaufbauenden Effekt}

- Aus In-vitro-Untersuchungen ist bekannt, dass Strontiumranelat (SR) neben seiner antiresorptiven Wirkung den Knochenaufbau aktiviert. Diese Eigenschaft konnte nun auch in einer großen vergleichenden Biopsie-Studie mit postmenopausalen Frauen dokumentiert werden.

In der internationalen Doppelblindstudie erhielten 268 betroffene Patientinnen randomisiert entweder 1 x täglich $2 \mathrm{~g}$ Strontiumranelat $\left(\right.$ Protelos $^{\oplus}$ ) oder $1 \mathrm{x}$ wöchentlich $70 \mathrm{mg}$ Alendronat. Um die Wirkung der Medikation auf den Knochenstoffwechsel zu dokumentieren, wurden zu Therapiebeginn sowie nach sechs bzw. zwölf Monaten Biopsien aus dem Beckenkamm entnommen und auf verschiedene Parameter des Knochenaufbaus untersucht.

Das wichtigste Kriterium für die Knochenneubildung - und damit auch pri- märer Endpunkt der Studie - war der prozentuale Anteil der mineralisierenden Oberfläche (MS/BS) an der Gesamtoberfläche des Knochens (BS), also der Anteil, an dem neuer mineralisierter Knochen angelagert wurde. Bereits nach sechs Monaten zeigte sich unter SR eine signifikant höhere Aktivität des Knochenaufbaus als in der Alendronat-Gruppe (MS/BS $=2,94 \%$ vs. $0,20 \%)$. Diese überlegene Wirksamkeit auf die Knochenbildung kam nach zwölf Monaten noch deutlicher zum Ausdruck.

Ein signifikanter Anstieg zeigte sich auch hinsichtlich der sekundären Zielparameter wie Appositionsrate des mineralisierten Knochens (MAR) und Knochenaufbaurate (BFR = MS x MAR). „Die Studie“, so Prof. Dieter Felsenberg, Berlin, „liefert erstmals harte Daten dafür, dass unter SR neuer Knochen produziert wurde." Hinweise darauf hatte schon die vorangegangene Head-to-Head-Studie geliefert, bei der die Knochenstruktur mittels hochauflösendem Mikro-CT unter die Lupe genommen wurde: Im Vergleich zu Alendronat zeigte sich eine deutliche Verbesserung der Mikroarchitektur sowie eine Zunahme der kortikalen Dicke und des Knochenvolumens.

Daten über mittlerweile zehn Jahre zeigen, dass SR auch langfristig gut vor vertebralen, nicht vertebralen und Hüftfrakturen schützt. In der offenen Verlängerung der Phase-III-Studien SOTI und TROPOS blieb die Frakturreduktion trotz des steigenden Altersrisikos auch über den Zeitraum von fünf Jahren hinaus erhalten.

\footnotetext{
- Dr. med. Martina-Jasmin Utzt

Quelle: Fortbildungsabend "Update Protelos Neueste Daten vom ECCEO", Nürnberg, März
} 2011 (Veranstalter: Servier) 